\section{Compliance with ethical standards}

Conflict of interest The authors declare that they have no conflict of interest.

\section{References}

1. Roberts DW, St Leger RJ. Metarhizium spp., cosmopolitan insectpathogenic fungi: mycological aspects. Adv Appl Microbiol. 2004;54:1-70.

2. Dorin J, Debourgogne A, Zaidi M, et al. First unusual case of keratitis in Europe due to the rare fungus Metarhizium anisopliae. Int J Med Microbiol. 2015;305:408-12.

3. De Garcia MC, Arboleda ML, Barraquer F, et al. Fungal keratitis caused by Metarhizium anisopliae var. anisopliae. J Med Vet Mycol. 1997;35:361-3.
4. Jani BR, Rinaldi MG, Reinhart WJ. An unusual case of fungal keratitis: Metarrhizium anisopliae. Cornea. 2001;20:765-8.

5. Motley WW, Melson AT, Mortensen JE. Pediatric Metarrhizium anisopliae keratitis. J AAPOS. 2011;15:101-3.

6. Amiel H, Chohan AB, Snibson GR, et al. Atypical fungal sclerokeratitis. Cornea. 2008;27:382-3.

7. Eguchi H, Toibana T, Hotta F, et al. Severe fungal sclerokeratitis caused by Metarhizium anisopliae: a case report and literature review. Mycoses. 2015;58:88-92.

8. Derhy D, Sauer A, Sabou M, et al. Surgical treatment of Metarhizium anisopliae sclerokeratitis and endophthalmitis. Indian $\mathbf{J}$ Ophthalmol. 2017;65:523-6.

9. Showail MJ, Kus JV, Tsui GK, et al. Fungal keratitis caused by Metarhizium anisopliae complex. Med Mycol Case Rep. 2017;17:28-30.

10. Thomas PA, Kaliamurthy J. Mycotic keratitis: epidemiology, diagnosis and management. Clin Microbiol Infect. 2013;19:210-20.

\title{
Reversible retinal toxicity in early oral Entecavir therapy for hepatitis-B infection
}

\author{
Suman Pilli ${ }^{1} \cdot$ Peck Lin Lip ${ }^{1}$
}

Received: 30 April 2018 / Accepted: 22 May 2018 / Published online: 22 June 2018

(c) The Royal College of Ophthalmologists 2018

We illustrate an acute retinal toxicity from oral Entecavir (Bristol-Myers-Squibb, USA) as treatment for acute hepatitis-B infection, and subsequent reversal of retinal toxicity upon stopping Entecavir with full restoration of visual functions.

\section{Case report}

A 52-year-old Caucasian male presented with unilateral sudden painless reduced vision and central scotoma of 5day duration. Patient was diagnosed acute hepatitis-B infection 6 weeks before and was started with oral

Peck Lin Lip

pllipwoo@gmail.com

1 Birmingham and Midland Eye Centre, Sandwell and West Birmingham Hospitals NHS Trust, City Hospital, Dudley Road, Birmingham B18 7QH, UK
Entecavir $0.5 \mathrm{mg}$ once daily. His presenting vision was 6/15OS and 6/6OD, normal pupillary reactions, full colour-perception and no ocular inflammation. Right fundus was normal while left showed mildly hyperaemic optic disc and a central well-demarcated hypopigmented area in posterior pole (Fig. 1: 1A). Both fundusfluorescein angiography (Fig. 1) and optical coherence tomography (Fig. 2) depicted corresponding retinal pigment epithelial (RPE) and photoreceptors abnormality. He had also established patchy central Goldmann field defects and grossly reduced full-field electroretinogram (delayed responses from both rod and cones) in OS only. Excluded retinovasculitis and satisfactory normal systemic investigations, a suspicion of drug toxicity was made. Entecavir was stopped immediately and remained off when his liver function test was also normalised. At 2month review, his vision had reached $6 / 6$, centre RPE hypopigmentation became indistinct. By 5 months, all visual function tests and fundoscopy were fully restored (Figs. 1, and 2). 

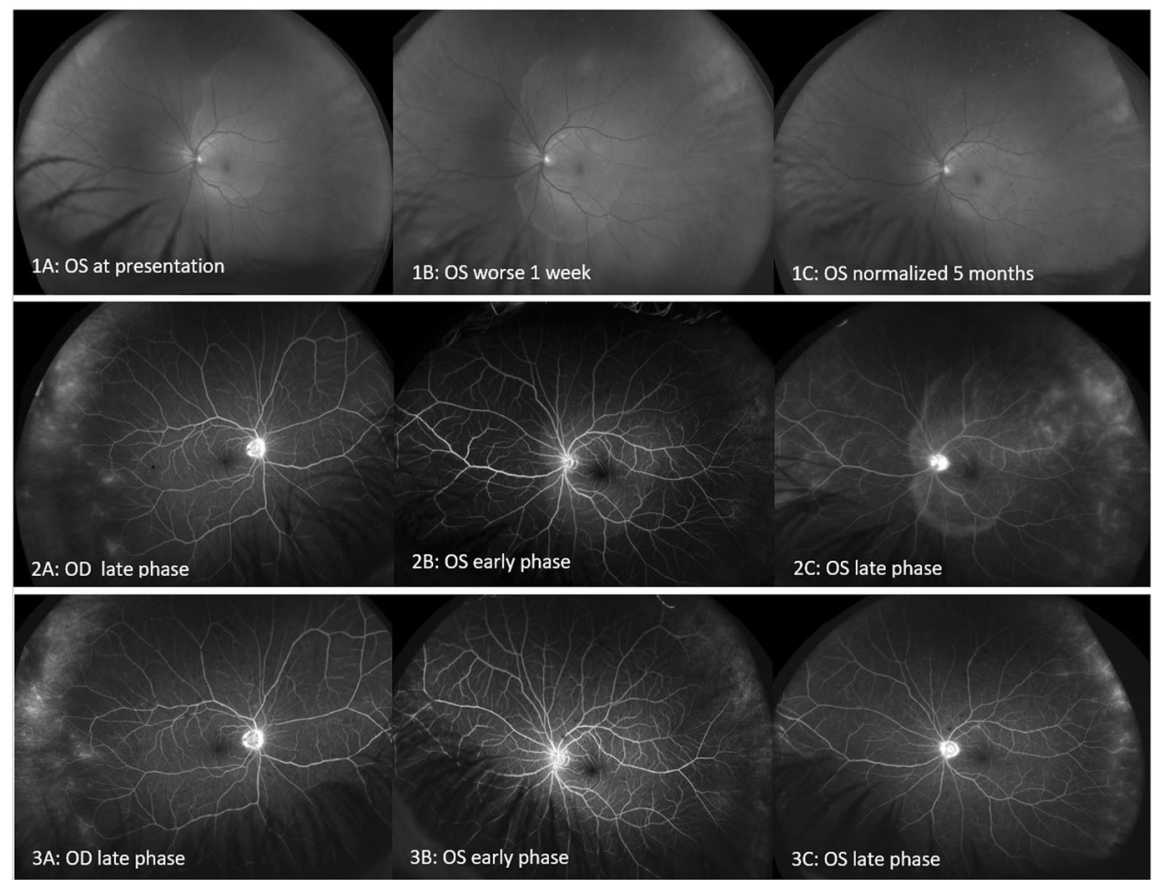

Fig. 1 Fundus photographs and fluorescein angiography (FFA). Fundus photographs of OS showed a clear demarcation of retinal pigment epithelial (RPE) hypopigmentation in posterior pole at presentation (1A). Patient's vision was 6/15 and complained of central scotoma, Entecavir was stopped at this point due to suspicion of retinal toxicity. At 1-week review, vision was worse (6/18 OS) and there was evidence of enlargement in demarcated RPE hypopigmentation area (1B). At 5-month review, patient had made a full spontaneous visual recovery in all aspects with 6/6 vision (1C). FFA at 1 week after presentation $(2 \mathrm{~A}-\mathrm{C})$ : OD showed late-phase temporal peripheral distal venous staining and peripapillary hyperfluorescence but normal posterior pole (2A). OS showed early well-demarcated area of early RPE layer hyperfluorescence corresponding to clinical hypopigmentation (2B), and remained staining in late phase (2C). There was more intense hyperfluorescence on optic disc and wider peripheral distal venous staining. FFA at 5 months after presentation $(3 \mathrm{~A}-\mathrm{C})$ : both eyes showed significant normalisation with some residual peripheral venous staining. $\mathrm{OS}=$ Left eye, $\mathrm{OD}=$ Right eye

Fig. 2 Optical coherence tomography (OCT) scans. At presentation, OCT showed normal retinal layers in OD (a), contrasting abnormal retinal layers in OS (b) with loss of reflectivity of photoreceptor ellipsoid zone and outer segments with intact external limiting membrane (white arrowhead), and multiple small high reflective elevations seen on RPE layer (white arrows). Repeated OCT at 5 months confirmed restoration of all retinal layers in OS (c). $\mathrm{OS}=$ Left eye, $\mathrm{OD}=$ Right eye

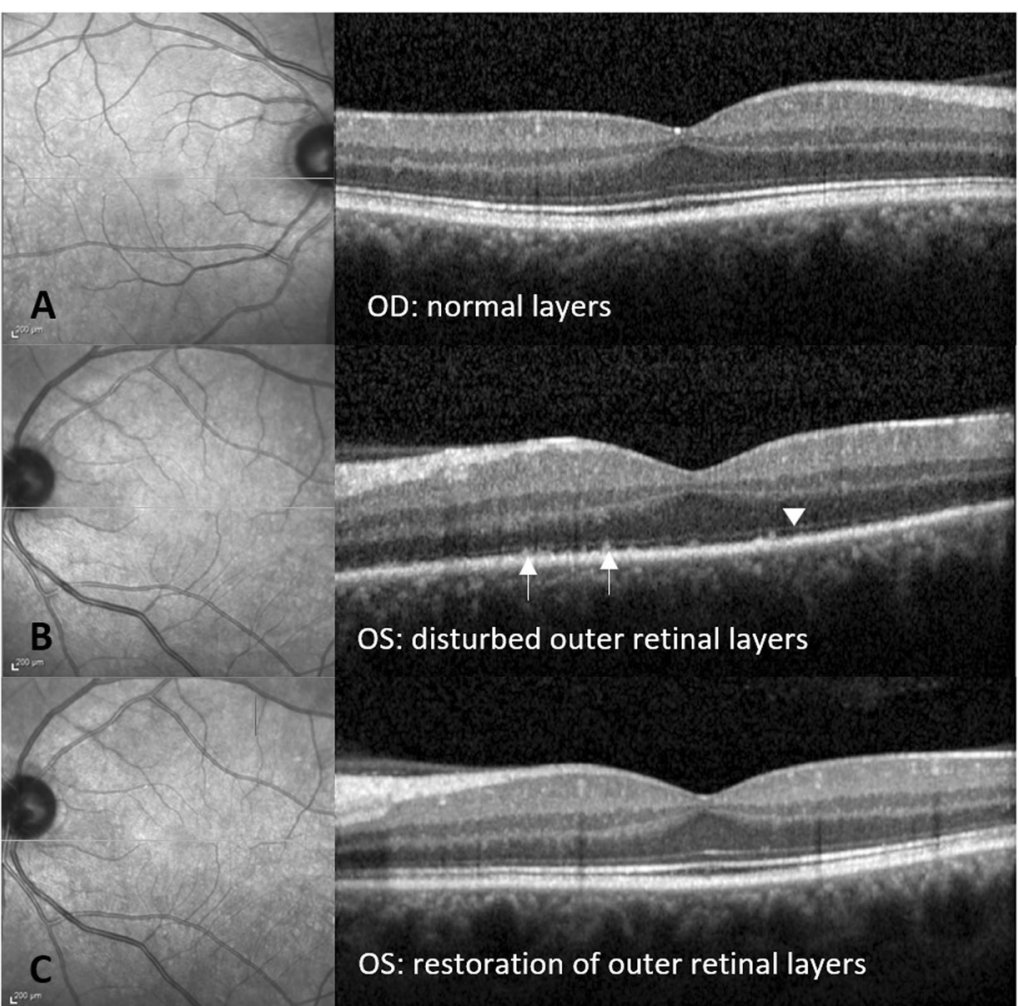


Entecavir is an anti-viral drug commonly used in treatment of hepatitis-B infection but there was no recognised ocular toxicity in its association [1-3]. The mechanism of retinal toxicity remains unclear with lacking histopathological studies. There was a published case report with irreversible blindness when on year(s) of Entecavir complicated by diabetic retinopathy at the time of suspected retinal toxicity [4]. Our case shared similar clinical features as their reported second eye. Notably, Entecavir drug toxicity is causing sequential rather than bilateral simultaneous eye manifestations. Our patient's left eye was profoundly involved but his right eye had not progressed more than the minimal peripheral vascular staining in late fundus photographs and fluorescein angiography phase (Fig. 1: 2A).

Our case confirmed short-term Entecavir use could cause reversible retinal toxicity upon drug cessation. Prescribing physicians and ophthalmologists should be aware of the potential retinal toxicity of Entecavir. We reported this adverse drug reaction to the UK Medicines and Health Products Regulatory Agency.
Acknowledgements We thanked Dr. Peter Good/Dr. Emma Berrow with their visual function team and Ms. Rosie Auld with her orthoptic team for their accommodative prompt services in performing/interpreting the results of visual function tests at various stages.

\section{Compliance with ethical standards}

Conflict of interest The authors declare that they have no conflict of interest.

\section{References}

1. Entecavir. National Center for Biotechnology Information. Pubchem compound database; $\mathrm{CID}=153941$. http://pubchem.ncbi.nlm. nih.gov/compound/153941.

2. Wong GL, Seto WK, Wong VW, Yuen MF, Chan HL. Review article: long-term safety of oral anti-viral treatment for chronic hepatitis-B. Aliment Pharmacol Ther. 2018;47:730-7.

3. Kayaaslan B, Guner R. Adverse effects of oral antiviral therapy in chronic hepatitis B. World J Hepatol. 2017;9:227-41.

4. Muqit MK, Stanga PE, Vilar FJ, Patton N. Presumed entecavirinduced ocular toxicity. Eye. 2011;25:1665-8.

\title{
Christmas tree cataract and myotonic dystrophy type 1
}

\author{
Dionysios Pagoulatos ${ }^{1} \cdot$ Zoi Kapsala $^{2} \cdot$ Olga E. Makri $^{1} \cdot$ Constantinos D. Georgakopoulos $^{1}$
}

Received: 14 March 2018 / Revised: 2 May 2018 / Accepted: 1 June 2018 / Published online: 9 July 2018

(c) The Royal College of Ophthalmologists 2018

We have read with great interest the recent article by Papadopoulos et al. [1] reporting early onset posterior subscapular cataract in 9 out of 28 patients with myotonic dystrophy type 2 (DM2). They also mentioned that this type of cataract was the first symptom in $7(25 \%)$ of the studied DM2 patients.

Dionysios Pagoulatos pagdion@gmail.com

1 Department of Ophthalmology, School of Medicine, University of Patras, Patras, Greece

2 Department of Ophthalmology, School of Medicine, University of Crete, Heraklion, Greece
Here, we would like to present our results regarding the incidence of Christmas tree cataract in patients with the most common form of DM, myotonic dystrophy type 1 (DM1). Christmas tree cataract is considered a characteristic finding in subjects with DM1. The retrospective review of medical records of 23 patients with DM1 revealed the presence of Christmas tree cataract in 13 patients $(56 \%)$. The multicoloured, iridescent lens opacities were unilateral in 10 out of the 13 patients and asymmetric bilateral in 3 patients. Age when cataract was diagnosed was $47 \pm 5$ years (range: $35-52$ years). The cataract was the first sign of the disease for 11 patients and was detected accidentally during a routine ophthalmological examination. Best corrected visual acuity was $0.06 \pm 0.08 \log$ MAR (range: -0.1 to $0.2 \operatorname{logMAR}$ ). The interval between diagnosis of cataract and DM1 was $10 \pm 2$ years (range: 